\section{La competencia comunicativa intercultural en cursos de inmersión en ELE. Una experiencia con estudiantes australianos en Colombia}

doi:Io.I I 44/Javeriana.SYP33-65.lcci
Intercultural communicative competence in SFL immersion courses, an experience with Australian students in Colombia

Competência comunicativa intercultural em cursos de imersão em ELE uma experiência com alunos australianos na Colômbia
Recibido: Mayo 5, 2014 Aceptado: Agosto 20, 2014

Submission date: May 5, 20I4 Acceptance date: August 20, 2014

\section{Origen del artículo}

Este artículo surge del trabajo conjunto de las investigadoras realizado antes, durante y después de un curso de inmersión en español como lengua extranjera, realizado en la Pontificia Universidad Javeriana. El curso de inmersión en ELE se empezó a preparar en enero de 2012 y se realizó entre el 18 y el 29 de noviembre del mismo año; en el primer semestre de 2013 se revisó toda la información recolectada sobre la experiencia y en junio de 2013 se inició la escritura del artículo.

Glenda Mejía

Australiana. PhD en Estudios Culturales. Profesora y coordinadora de estudios de español, coordinadora del Programa en Lenguajes y directora del Centro de Recursos de Español en el Royal Melbourne Institute of Technology (RMIT).Correo electrónico: glenda.mejia@rmit.edu.au

Nancy Agray-Vargas

Magíster y candidata a doctora en lingüística aplicada a la enseñanza de español como lengua extranjera. Profesora asociada, Directora de la Maestría en Lingüística Aplicada del Español como Lengua Extranjera, de la Pontificia Universidad Javeriana, Departamento de Lenguas, Facultad de Comunicación y Lenguaje. Correo electrónico: nagray@javeriana.edu.co 


\section{Resumen}

El objetivo de este artículo es presentar la organización y realización de un curso de inmersión de español como lengua extranjera (ELE) por parte del Royal Melbourne Institute of Technology (RMIT), de Australia, y la Pontificia Universidad Javeriana (PUJ), de Bogotá, Colombia. El curso fue realizado durante dos semanas, en noviembre de 2012, y en él participaron diez estudiantes australianos.

Se presenta la manera como se realizó la experiencia y sus resultados, entre los cuales se destacan el mejoramiento en la competencia comunicativa y en la competencia cultural e intercultural de los estudiantes, a propósito de haber trabajado la lengua y la cultura como aspectos intrínsecamente relacionados. Se concluye que esta modalidad de cursos permite un mayor desarrollo lingüístico y también un aprendizaje cultural e intercultural invaluable para el estudiante, en el proceso de adquisición de la denominada competencia comunicativa intercultural.

Palabras clave: cursos cortos; inmersiones en lengua extranjera; competencia comunicativa intercultural; ELE; español

\section{Abstract}

The aim of this paper is to present the organization and implementation of an immersion course in Spanish as a foreign language (SFL) from the Royal Melbourne Institute of Technology (RMIT) of Australia, and the Pontificia Universidad Javeriana (PUJ), Bogota, Colombia. The course was conducted over two weeks in November 20I2, and Io Australian students participated. We present the experience and its results, among which we highlight the improvement in communicative competences, as well as cultural and intercultural competences of students, especially for having worked aspects intrinsically related such as language and culture. It is concluded that this type of courses allow a greater linguistic development and an invaluable cultural and intercultural learning for students that are in the acquisition process of the so called intercultural communicative competence. Keywords: short courses; immersion in a foreign language; intercultural communication competence; SFL; Spanish

\section{Resumo}

O objetivo deste artigo é apresentar a organização e realização de um curso de imersão de espanhol como língua estrangeira (ELE) por parte do Royal Melbourne Institute of Technology (RMIT), da Austrália, e a Pontifícia Universidade Javeriana (PUJ), de Bogotá, Colômbia. O curso foi realizado durante duas semanas, em novembro de 20I2, e nele participaram io alunos australianos. Apresenta-se a maneira como a experiência foi feita e seus resultados, entre os quais destacam-se o aperfeiçoamento na competência comunicativa e na competência cultural e intercultural dos alunos, a propósito de ter trabalhado a língua e a cultura como aspectos intrinsecamente relacionados. Conclui-se que este tipo de curso permite maior desenvolvimento linguístico e mesmo uma aprendizagem cultural e intercultural inestimável para o aluno, no processo de aquisição da nomeada competência comunicativa intercultural.

Palavras-chave: cursos de curta duração; imersão em língua estrangeira; competência comunicativa intercultural; ELE; espanhol 
Glenda Mejía

Nancy Agray-Vargas

\section{La competencia comunicativa intercultural en cursos de inmersión en ELE. Una experiencia con estudiantes australianos en Colombia}

\section{Introducción}

Actualmente, uno de los propósitos en el nivel universitario en todo el mundo es despertar en los estudiantes una conciencia global que les permita incrementar el conocimiento de aspectos sociales, económicos, políticos y culturales de otros países; tener la oportunidad de sensibilizarse y de aprender acerca de otras formas de ver el mundo; conocer otras opiniones, y ser conscientes de la existencia de otras culturas. Igualmente, se espera que puedan alcanzar una mayor competencia comunicativa en una lengua extranjera, experimentar un crecimiento personal y desarrollar destrezas para su futura vida profesional. En otras palabras, formar ciudadanos del mundo.

Diversos estudios han demostrado que estudiar en el extranjero beneficia a los estudiantes, ya que les proporciona un conocimiento general, un cambio de actitud acerca del mundo (DeLoach, Saliba, Smith, \& Tiemann, 2003) y un conocimiento transcultural (Bakalis \& Joiner, 2004; Douglas \& Jones-Rikkers, 2001; Kitsantas, 2004; Kitsantas \& Meyers, 200I). Asimismo, estudios como el de Collentine y Freed (2004) han comprobado que los cursos cortos de inmersión son más efectivos que los intercambios de estancia.

Teniendo en cuenta lo anterior, el Royal Melbourne Institute of Technology (RMIT), de Australia, decidió avanzar en este camino y buscar instituciones "socias" que compartan las mismas inquietudes e intereses. Así es como llegó a la Pontificia Universidad Javeriana, de Bogotá, institución con la que empezó a trabajar.

El fundamento de este trabajo conjunto es la concepción compartida por las dos instituciones de propiciar en los estudiantes la conciencia de un mundo global, la necesidad de comprender las culturas propias y ajenas, el respeto por la diferencia y el aprendizaje de estos valores en la acción.

Experiencias como esta pueden servir de referencia para que en otros contextos se evalúe la viabilidad de los programas de inmersión y se posibilite el intercambio estudiantil y profesoral, de manera que, efectivamente, contribuyamos a la construcción de una sociedad más plural, diversa y respetuosa de las diferencias a partir de las comunidades académicas.

\section{La política del RMIT como pasaporte global} y la política de internacionalización de la PUJ

El RMIT tiene como objetivo crear una visión global de pensamiento, actitud y acción en sus estudiantes, que les permita aprender la complejidad del mundo contemporáneo y tener la oportunidad de trabajar y 
entablar experiencias interculturales. También tiene una visión de conexión a través de actividades profesionales en colaboración con instituciones, industrias y organizaciones que apoyen e impacten en nuestra educación e investigación. En consonancia con esta visión, la rectora Margaret Gardner (2010), en el documento del plan estratégico del RMIT 20II2015, Where Bold Hopes Can Be Realised, manifiesta:

Como universidad tecnológica y de diseño, RMIT no solo se enfoca en su educación e investigación, sino que también se compromete con una filosofía basada en la acción y la experiencia. Esto requiere que la educación y la investigación se apoyen en la asociación y el trabajo con otros que nos ayuden a alcanzar nuestras metas. [...] Debemos empezar con nuestros estudiantes y con el compromiso de transformar su futuro, no importa dónde ni cómo empiecen ${ }^{\mathrm{I}}$.

Por su parte, la Pontificia Universidad Javeriana, en su Política de Internacionalización, señala que entre las intencionalidades con las cuales la adopta dicha política se encuentra:

Propiciar una conciencia global e intercultural, y el desarrollo de las comprensiones de las culturas propias y ajenas, fundamentados en la interdependencia entre los pueblos y las sociedades y el respeto al pluralismo, bases de la solidaridad, de la convivencia pacífica entre las naciones y de la auténtica ciudadanía global².

Con base en estas premisas, en la política del RMIT de internacionalizar sus cursos y dar la oportunidad a sus estudiantes de conocer otros países y culturas, la coordinadora del Programa de Español del RMIT contactó a la PUJ para establecer la viabilidad de realizar un curso de inmersión de español, teniendo en cuenta que entre las dos instituciones hay un convenio de cooperación in-terinstitucional. Dicho convenio busca promover los estudios de intercambio que le permiten al estudiante desarrollar no solo una experiencia de conocimiento en su área de estudio, sino también una experiencia cultural. Esta iniciativa fue bien recibida en la PUJ y así empezó el proceso de creación del primer curso de inmersión de español del RMIT, en asocio con la PUJ.

\section{Algunos conceptos de base}

El diseño de los cursos de lengua extranjera en la modalidad de inmersión, es decir, los que se desarrollan en el contexto en el cual la lengua es 
vehículo de comunicación en los ámbitos sociales y privados, requieren, por una parte, la identificación de los intereses y necesidades de quienes se deciden a viajar a otro país a estudiar la lengua extranjera $y$, por la otra, tener una perspectiva conceptual que permita sustentar coherentemente la propuesta del curso de inmersión que se va a ofrecer.

En ese sentido, lo que se detecta en primer lugar en quienes desean realizar un curso de inmersión, más allá del interés puramente técnico de aprender o consolidar algunos aspectos lingüísticos y mejorar el nivel de lengua, es un deseo de vivir una experiencia cultural. Esto significa conocer a otros, sus costumbres, creencias y comportamientos cotidianos y relacionarse con ellos, no solo porque la situación misma así lo exige, sino también porque, como seres sociales que somos, sentimos la necesidad de interactuar con los demás y la curiosidad por todo aquello que es "diferente". Por ello, para un curso de esta naturaleza es necesario establecer cuidadosamente el concepto de cultura y la relación lengua-cultura que se debe manejar, así como las competencias que se deben desarrollar en los estudiantes, tanto dentro como fuera de las aulas de clase.

En relación con el concepto cultura, este ha sido definido por investigadores de distintas disciplinas. Así, por ejemplo, algunas de las teorías de comunicación intercultural lo definen y relacionan con los valores, creencias y comportamientos de un grupo de personas (Gudykunst, 2004; Lustig \& Koester, 2006, citado en Jackson, 2010). Por su parte, en enseñanza de lenguas, dependiendo de la corriente metodológica, se pueden identificar diferentes concepciones de cultura, entre las que se destacan: en la corriente tradicional, la que se relaciona con la llamada "alta cultura", que es una de las que más han perdurado en la enseñanza de lenguas extranjeras. En ella se entiende la cultura como "la suma de las grandes ideas representadas en las grandes obras clásicas de la literatura, pintura, música y filosofía" (Hall, I997, p. 2)3. En las corrientes nociofuncionales el concepto presente es el de la "baja cultura", entendido como "las formas ampliamente distribuidas de la música popular, la publicidad, el arte, el diseño y la literatura o de las actividades del tiempo libre y del entretenimiento que hacen la vida cotidiana de la mayoría de la 'gente popular"' (Hall, I997, p. 2) ${ }^{4}$. Y en las corrientes metodológicas comunicativas, más allá de una concepción de cultura, se encuentra una de interculturalidad en procesos comunicativos, dado que el objetivo de incluir el componente cultural en la enseñanza de una lengua extranjera es ayudar al estudiante a que se oriente en una cultura diferente a partir de la propia, y a que utilice y aprenda estrategias de la comunicación intercultural (Universidad Nebrija, I998). Esta última concepción es la que nos interesa en el caso de esta experiencia.

En la perspectiva comunicativa, los contenidos que se trabajan están relacionados con grandes categorías de la sociología, como la familia y el país, con el fin de llevar al estudiante a que establezca comparaciones, intentando no hacer juicios de valor entre sus representaciones mentales y su sistema de valores con los de la cultura de llegada (en este caso, la cultura colombiana). Las actividades tienen la finalidad de que el estudiante "se ejercite" en el "autodescubrimiento" y la percepción de hechos culturales y en su comparación no valorativa (Agray, 20I0, p. 30).

Esta manera de entender la presencia del componente cultural en la enseñanza de lenguas es la que nos lleva a proponer y tener como meta en los cursos de español como lengua extranjera en la PUJ, el desarrollo de la competencia comunicativa intercultural. En el aprendizaje de una lengua extranjera no basta con conocer acerca de la "alta" y "baja" cultura del país y de los hablantes de la lengua meta. Nos interesa la formación integral de los estudiantes y ayudarlos a convertirse en ciudadanos del mundo, a entender la interculturalidad en la práctica de la interacción entre culturas, en el intercambio y la comunicación en las que se respeta la diversidad cultural desde los principios de igualdad y reconocimiento del otro, como un espacio de colaboración para la producción conjunta de conocimiento, valores, discursos y sentido en un contexto común (Agray, 20Io, p. 34); en esta experiencia, el contexto de Bogotá, Colombia y la PUJ. 
El concepto de competencia comunicativa intercultural también ha sido definido desde diferentes perspectivas, pero para efectos de este artículo podemos decir, simplemente, siguiendo a Fantini (200o, p. 3), que hay acuerdo en que su naturaleza es de "doble vía". Es decir, "el desarrollo de la competencia en otra cultura y la competencia en su lengua da la oportunidad de reflexionar profundamente sobre la propia” (Fantini, 200o, p. 3). Asimismo, el desarrollo de esta competencia está muy relacionado con la toma de decisiones personales: el progreso y adaptación a una segunda cultura depende de lo que cada uno disponga para ello. La decisión individual puede ir desde el rechazo de la cultura meta hasta el ajuste cultural superficial y algunas veces profundo (Fantini, 2000, p. 3).

Por ello, el desarrollo de la competencia comunicativa intercultural se propone como un reto y una posibilidad para docentes y estudiantes, puesto que ofrece la posibilidad de trascender la visión limitada del mundo propio. Quien no ha experimentado otra cultura o no ha intentado comunicarse por medio de otra lengua, no es generalmente consciente del medio en el que ha vivido (Fantini, citado por Agray, 2010, p. 37). El anterior reto es el que decidieron tomar los estudiantes australianos al viajar a Colombia.

\section{Preparación de la experiencia}

La preparación tuvo lugar durante el año 2012 y se realizó en los dos países, Australia y Colombia, con la participación y el trabajo conjunto de las coordinadoras y de la Oficina de Relaciones Interinstitucionales de la PUJ, tanto en los aspectos administrativos y logísticos, como en los académicos. En Australia, la preparación administrativa incluyó, entre otros aspectos: la consecución de recursos, cuyo resultado fue que parte de la inmersión en Colombia estuvo subvencionada con una beca del Departamento de Educación del gobierno australiano (Department of Education, Employment and Workplace Relations [DEEWR]) y el resto fue cubierto por los estudiantes; y la acreditación del curso como parte de los estudios de español, integrado a los estudios de licenciatura en el RMIT. En Colombia, desde la Oficina de Relaciones Interinstitucionales de la PUJ, las actividades administrativas estuvieron relacionadas con el ajuste presupuestal para facilitar la experiencia; la consecución de familias para la estadía de los estudiantes y la coordinación de diferentes instancias de la Universidad para la organización de las salidas culturales, la alimentación y el transporte de los estudiantes. Vale la pena destacar el trabajo realizado con el grupo estudiantil Out of Town, dedicado a acompañar la experiencia en los aspectos culturales.

En cuanto a lo académico, en el RMIT los estudiantes realizaron, un semestre antes de la inmersión, tres tipos de actividades. Por una parte, estudiaron, de manera general, diferentes aspectos culturales de Hispanoamérica, como la comida y la cortesía. Aunque no se centraron en Colombia, los estudiantes fueron informados acerca de que estos aspectos también forman parte de la cultura colombiana. Por la otra, los estudiantes asistieron a un taller de comunicación intercultural creado por la profesora de español y una estudiante colombiana de la PUJ que estaba en Australia como estudiante internacional del RMIT. El propósito del curso fue concientizar a los estudiantes de los "Do and Don't" en Colombia y acercarlos al conocimiento del ambiente, la comida y la cortesía en el país, puesto que iban a tener la experiencia de estudiar y vivir con una familia colombiana. Por último, la profesora a cargo del curso trabajó para que los estudiantes comprendieran que, al estar en un país en el que no se habla su lengua, es normal cometer errores gramaticales, pero es importante tratar de no incurrir en errores culturales, lo cual se puede lograr conociendo y estando dispuestos a entender las diferencias culturales, de actitudes, comportamientos y valores.

En el caso de la PUJ, igualmente se realizaron tres actividades académicas durante un semestre: primero, preparar diferentes versiones del programa del curso, teniendo en cuenta el nivel de lengua de los estudiantes, sus características, 
intereses, necesidades y las necesidades de la Institución (RMIT), en relación con la experiencia. Dichos programas fueron revisados y retroalimentados por la coordinadora del RMIT hasta que se consolidó la versión final. Segundo, trabajar con las profesoras a cargo en la preparación del curso de inmersión: conocimiento de aspectos culturales de Australia y las características de los estudiantes, para definir detalladamente las actividades que se iban a desarrollar. Tercero, preparar a un grupo de estudiantes de la Licenciatura en Lenguas Modernas que cursaban el Énfasis en Enseñanza de ELE para trabajar como monitores de los estudiantes australianos, en una jornada de estudio previa al examen final del curso.

Con la anterior preparación y teniendo en mente que los estudiantes tuvieran la experiencia de aprender la lengua española y la cultura colombiana de primera mano por estar viviendo en casas de familias colombianas e interactuando con estudiantes colombianos, se desarrolló el programa.

\section{Dos semanas en Colombia}

\section{Participantes}

Como ya se mencionó, en esta experiencia de un curso de inmersión en español como lengua extranjera en la PUJ participaron diez estudiantes australianos en su segundo año de carrera del RMIT. Cinco hombres y cinco mujeres en edades entre 20 y 26 años. De los diez estudiantes, nueve eran de la Escuela de Estudios de Relaciones Internacionales y uno de la Escuela de Ciencias, en el área de Ingeniería. Estos estudiantes habían completado el nivel A2, de acuerdo con el Marco Común de Referencia Europeo (MCRE), y el curso en Colombia era el nivel Bi.I, según el mismo MCRE, que corresponde, en la escala de la PUJ, al nivel 5. Sin embargo, en la competencia oral el nivel no era el mismo; algunos estudiantes tenían un nivel de competencia mayor y más fluidez comunicativa y no tenían ninguna actitud negativa al hablar.

\section{Materiales}

Además de los materiales propios del desarrollo de las diferentes actividades del curso, incluidos los exámenes finales (escrito y oral), los materiales utilizados en esta experiencia fueron: el programa del curso como carta de navegación con una organización detallada de actividades por horas y días, que se fue ajustando en el desarrollo de la experiencia. Un cuestionario de retroalimentación del programa de ELE, con el objetivo de ajustarlo a este grupo en particular, y un cuestionario final de evaluación de toda la experiencia, realizado por la Oficina de Relaciones Interinstitucionales de la PUJ. Además, a los estudiantes se les dio la oportunidad de expresar verbalmente su estado de satisfacción y comodidad con el curso, actividad que no solo sirvió para cualificar su desarrollo, sino también como insumo importante para próximas experiencias.

La coordinadora del RMIT realizó una observación participativa cada día durante las dos semanas. Estas observaciones se llevaron a cabo a la hora del almuerzo, en las clases, en los eventos culturales y excursiones y en el transporte público. También se realizaron entrevistas informales diarias, individuales y en grupo, después de clase, con el fin de brindar apoyo emocional, estimular la reflexión sobre la experiencia cultural y motivar a los estudiantes que no querían hablar para que compartieran experiencias, problemas en común y prácticas para abordar los problemas. Las preguntas realizadas en estas entrevistas fueron organizadas en cuatro temas que sirvieron para la evaluación de la experiencia. Dichos temas fueron:

- La experiencia de vivir con una familia colombiana.

- La metodología del curso de español y las profesoras.

- La participación en las actividades culturales y de estudio y el apoyo de los estudiantes colombianos en ellas.

- Los comentarios generales y sugerencias en relación con toda la experiencia del curso de inmersión. 


\section{Desarrollo}

El curso fue diseñado bajo la metodología del enfoque por tareas, que promueve el aprendizaje significativo de la lengua al relacionar las experiencias de comunicación cotidianas con el contexto de enseñanza y aprendizaje. Al igual que en los demás cursos de ELE que se dictan en la PUJ, en esta experiencia la lengua de enseñanza y de comunicación tanto dentro como fuera del aula fue solamente el español.

Durante el curso, los estudiantes recibieron entre cinco y seis horas diarias de clase de ELE e hicieron salidas culturales ligadas a los temas de estudio para facilitar su aprendizaje y práctica en contextos reales. Por ejemplo, visitaron lugares como el Museo del Oro, el Museo Botero, el barrio La Candelaria, el cerro de Monserrate, la Mina de Sal de Nemocón y una finca cafetera. En estos lugares los estudiantes realizaban actividades que luego debían reportar de diferentes formas en la clase de ELE, tales como relatar experiencias, contar chistes, recomendar, explicar procedimientos, etc. El logro de los objetivos propuestos y las competencias adquiridas en la inmersión fueron evaluados de manera formal con un examen escrito y otro oral; este último, mediante la presentación de un proyecto que los estudiantes fueron desarrollando durante su estadía en Colombia.

Igualmente, vivieron con familias colombianas, hicieron uso del transporte público para ir de su lugar de vivienda a la Universidad y viceversa; departieron con estudiantes colombianos de la PUJ en diferentes lugares, tales como discotecas, restaurantes, centros comerciales y parques, luego de completar la jornada de actividades académicas y culturales en la Universidad. La experiencia de vivir con una familia tenía como propósito que los estudiantes practicaran su español, observaran y aprendieran más de los aspectos culturales de Colombia y vivenciaran la cotidianidad de una familia y de un estudiante en este país. La coordinadora del RMIT estableció como regla hacer uso del español siempre, por lo menos en su presencia. El objetivo de esta regla era animar a los estudian- tes a aprovechar al máximo la oportunidad de comunicarse en español en todos los contextos para mejorar su competencia comunicativa y su léxico.

Como ya se señaló, después de las clases, la profesora del programa del RMIT se reunía con el grupo de estudiantes en un ambiente relajado que permitiera la reflexión cultural e intercultural de la experiencia, así como descubrir los posibles problemas individuales y colectivos en los distintos contextos, establecer el grado de satisfacción de los estudiantes y el nivel de aprendizaje de español. Luego de la reunión diaria los estudiantes tenían tiempo libre para explorar la ciudad, compartir con sus nuevos amigos colombianos o con su familia, o simplemente descansar.

\section{Resultados}

Los resultados de este curso de inmersión de dos semanas con estudiantes australianos del RMIT provienen de los siguientes instrumentos y actividades de recolección de información:

- Cuestionarios de evaluación del curso aplicados por la coordinadora del programa de ELE y por la Oficina de Asuntos Interinstitucionales de la PUJ.

- Entrevistas realizadas a los estudiantes por parte de la coordinadora del RMIT.

- Información suministrada por las profesoras y los monitores del curso.

- Comentarios generales y sugerencias de los estudiantes en relación con toda la experiencia del curso de inmersión.

- Exámenes finales presentados por los estudiantes.

\section{La experiencia de vivir con una familia colombiana}

Todos los estudiantes participantes afirmaron que la experiencia de vivir con familias colombianas fue muy positiva y que aprendieron nuevas y distintas formas culturales y sociales que no habían tenido la oportunidad de experimentar, aunque ya habían escuchado de ellas en los medios de comunicación 
(radio, televisión, internet, telenovelas, películas) y en su clase de español.

Algunos comentarios en los cuestionarios con respecto a lo que más les gustó de la experiencia y la valoración del alojamiento con las familias fueron: "La familia bogotana y la hospitalidad de la gente" "Vivir con una familia colombiana para mejor aprender español y la cultura", "Muchas cosas, particularmente la oportunidad de vivir una experiencia Bogotana con una familia colombiana", "Mi familia son los mejores".

Los anteriores comentarios demuestran que la adaptación de los estudiantes al entorno sociocultural colombiano fue positiva, a pesar de una imagen ya formada antes de llegar a Colombia, y corroboran lo encontrado en algunos estudios en relación con que el concepto e imagen de la nueva cultura también varía y depende de la experiencia de cada estudiante (Brecht, Davidson \& Ginsberg, 1993). Aunque la experiencia con las familias fue evaluada como positiva, algunos estudiantes hicieron comentarios en relación con las nuevas formas y experiencias culturales y sociales que descubrieron con su familia colombiana que, al principio, les parecieron muy diferentes a la manera propia de hacer las cosas, pero que después, aunque no las aceptaran, aprendieron a respetar. Un ejemplo de lo anterior es lo mencionado por tres estudiantes: "I don't like how the maid does everything for me, I feel very uncomfortable" ("No me gusta que la empleada del servicio me haga todo, me siento incómodo"). "Wow, today my host Mum brought me breakfast on bed” (“¡Guao!, hoy mi 'mamá anfitriona’ me llevó el desayuno a la cama"). "The maid cooks for me every single day" ("La empleada del servicio cocina para mí todos los días"). Algunos hicieron comparaciones con la película mexicana $Y$ tu mamá también (Alfonso Cuarón, 200I), específicamente con la escena en la que uno de los protagonistas no contesta el teléfono, a pesar de tenerlo junto a él, sino que deja que la empleada lo conteste. Estas situaciones podrían parecer insignificantes para un estudiante colombiano, pero no lo son para un estudiante australiano que vive en una sociedad en la que los estratos sociales no son tan marcados, no es común tener empleadas del servicio, o en una sociedad en la que los estudiantes son muy independientes y a los i8 años ya no viven con sus padres.

Lo comentado por los estudiantes corrobora lo que señalan DeLoach, et al. (2003) acerca del cambio de actitud sobre el mundo que se produce en los estudiantes a propósito de vivir la experiencia de un curso de inmersión. También lo mencionado por Fantini (2000) en cuanto a que el desarrollo de la competencia intercultural está relacionado con una decisión personal y con el progreso y adaptación a una segunda cultura. Algo que al principio les parecía inaceptable a algunos, después fue aceptado y respetado como otra manera de establecer relaciones en la cultura colombiana.

Algunos estudiantes también comentaron que el hecho de haber vivido con una familia colombiana les dio la oportunidad de practicar más su español y de hablar de otras cosas sobre las cuales no habrían tenido la oportunidad de hacerlo en clase o en un ambiente formal. Es decir, vivenciaron la denominada "baja cultura" al experimentar y realizar actividades de la vida cotidiana de la gente colombiana. Esta posibilidad motivaba a los estudiantes australianos a seguir practicando español y aprendiendo sobre aspectos culturales de Colombia, a pesar de tener jornadas largas de clase (entre cinco y seis horas diarias). Con ello se demuestra que esta forma tan directa de compartir con nativos es una ventaja significativa en comparación con permanecer en un hotel durante un curso de inmersión. Estudios como el de García y Bejarano (2008) han demostrado que algunos estudiantes, después de muchas horas de estudio, se sienten cansados y regresan al hotel, perdiendo así la oportunidad de interactuar con hablantes nativos del país de destino.

\section{El curso de español}

Todos los estudiantes afirmaron que se alcanzaron los objetivos propuestos en el curso, que su nivel de español mejoró y que sus expectativas se cumplieron. Esta percepción de avance en la competencia comunicativa de los estudiantes fue comprobada 
por las profesoras a lo largo del curso y con los resultados de los exámenes: todos aprobaron con calificaciones muy buenas, buenas y regulares. Igualmente, las observaciones de las interacciones de los estudiantes, realizadas por la coordinadora del RMIT, permitieron comprobar un mejor desempeño en la producción oral y escrita, y el logro de los objetivos de aprendizaje propuestos tanto por la PUJ como por el RMIT, tales como: escribir y comunicarse oralmente con un nivel de español razonable y efectivo en situaciones familiares, evaluar y debatir temas sociales y contemporáneos de la cultura hispana y compararlos con el contexto australiano, usar las estrategias de aprendizaje que se han adquirido y utilizarlas para demostrar un aprendizaje independiente.

De igual forma, tanto las profesoras del curso como la coordinadora del RMIT observaron el incremento especialmente en el léxico de los estudiantes al expresarse en español, en comparación con el primer día de clase y con el nivel de español que cada estudiante traía consigo (la profesora del RMIT lo conocía, ya que impartió la clase en el primer semestre de 2012). Por supuesto que, como ya se señaló, no todos alcanzaron el mismo nivel, unos mejoraron más que otros, pero en todos sí se evidenció un aumento en su competencia comunicativa.

El desarrollo en la competencia comunicativa intercultural de los estudiantes también fue comprobado en la presentación oral que realizaron el último día de clase, como examen oral. En esta presentación los estudiantes utilizaron de manera adecuada la gramática y el léxico aprendidos en clase, incluyeron aspectos de la cultura colombiana, como las obras de Gabriel García Márquez y de Fernando Botero o el sistema Transmilenio, y fueron capaces de expresar su opinión con respecto a conflictos de la sociedad colombiana, como la situación política entre la guerrilla y el gobierno colombiano.

De acuerdo con lo señalado en los conceptos de base, con lo realizado en la experiencia al parecer se propició que los estudiantes se orientaran en la cultura colombiana a partir de la propia y aprendieran y utilizaran estrategias de la comuni- cación intercultural (Universidad Nebrija, 1998). El proyecto que cada estudiante desarrolló durante toda su estadía y que presentó en la actividad final permitió que el estudiante "se ejercitara" en el "autodescubrimiento" y la percepción de hechos culturales y en su comparación no valorativa (Agray, 2010).

De la misma forma, en relación con el curso los estudiantes resaltaron la metodología, las actividades, la jornada de estudio previa a los exámenes con los estudiantes de la Licenciatura en Lenguas Modernas de la PUJ y la calidad de las docentes.

Tanto los estudiantes australianos como los estudiantes colombianos de la Licenciatura en Lenguas Modernas valoraron positivamente la jornada de estudio realizada antes de los exámenes finales. A los australianos les permitió practicar español, repasar para los temas, reforzar lo aprendido en clase y aclarar las dudas gramaticales. Algunos comentarios fueron "Sí fue bien, antes del examen podía mejorar cosas que no eran claras", "Muy útil, los estudiantes fueron muy útiles". Y a los estudiantes colombianos les permitió poner en práctica los conocimientos adquiridos en su formación como profesores de ELE e interactuar con los australianos. Al respecto, los estudiantes de la Licenciatura mencionaron haber sentido una gran responsabilidad y luego una gran satisfacción de haberles "ayudado" a estudiar a sus compañeros australianos; también afirmaron que la experiencia les había servido para dimensionar el reto que significa ser docentes de ELE.

Los resultados positivos de esta actividad confirman lo mencionado en los conceptos de base, acerca del interés por la formación integral de los estudiantes, por ayudarlos a convertirse en ciudadanos del mundo y a entender la interculturalidad en la práctica de la interacción (Agray, 20I0).

En el intercambio y la comunicación que se dio entre los estudiantes, ellos pudieron vivir la diversidad cultural desde los principios de igualdad y reconocimiento del otro, como un espacio de colaboración para la producción conjunta de conocimiento, valores, discursos y sentido en un contexto común. 


\section{Las actividades culturales y la participación de estudiantes colombianos}

En relación con este aspecto, encontramos que a los estudiantes les gustaron todas las actividades culturales y sociales programadas. La actividad mejor recibida y que más gustó fue la clase de baile de salsa porque el grupo de estudiantes y de bailarines la hicieron muy divertida y porque fue realizada por jóvenes de su misma edad. Igualmente porque para la mayoría de los estudiantes australianos, esta era la primera vez que bailaban con colombianos y también era la primera experiencia de participar en un baile "folclórico". Algunos comentarios: "Para mí la clase de baile fue la mejor experiencia, normalmente no me gusta bailar pero los bailarines y los estudiantes hicieron la experiencia muy divertida" "Toda era fantástica, la presentación de los bailarines y después la clase era perfecto porque me gusta bailar salsa mucho".

Asimismo, para los estudiantes australianos fue una experiencia extraordinaria poder hablar de temas de jóvenes con gente de su misma edad y poder jugar "pictionario" en español. Con respecto al grupo Out of Town, los estudiantes australianos resaltaron su amabilidad y el valor de tenerlos en la experiencia de la inmersión: "Fueron muy agradables", "Todos son muy agradables y esencial por la experiencia", "Los mejores". Contar con la participación de estudiantes colombianos en la inmersión les permitió a los estudiantes australianos entrar en contacto con variantes de la lengua y la cultura más modernas y más cercanas a ellos, si tenemos en cuenta que los jóvenes utilizan expresiones y manejan aspectos culturales que los profesores y la gente de más edad no utilizan o no conocen. Al final de la experiencia los estudiantes australianos ya usaban, en situaciones cotidianas, un léxico más informal y apropiado para los jóvenes, que habían ido adquiriendo con sus compañeros colombianos. Igualmente, los colombianos aprendieron aspectos del inglés y de la cultura de los jóvenes en Australia. Esto corrobora lo señalado al inicio del artículo, en relación con que, como seres sociales que somos, sentimos la necesidad de interactuar con los demás y la curiosidad por todo aquello que es "diferente", lo cual enriquece los procesos de aprendizaje.

\section{Comentarios y sugerencias}

En cuanto a la seguridad y la situación en Colombia, los estudiantes mencionaron que habían cambiado su punto de vista y que se habían sentido seguros. El hecho de haber usado el transporte público para movilizarse verifica estos comentarios: "Estaba preocupado en Australia por viajar a Colombia pero en dos semanas ya me siento cómodo y en casa". Con este resultado se corrobora lo señalado por Brecht et al. (I993) acerca de que el concepto e imagen de la nueva cultura también varía a propósito de la inmersión, como sucedió en este caso, pero depende de la decisión que tome el estudiante y de su flexibilidad ante la nueva experiencia cultural (Fantini, 2000).

Igualmente, los estudiantes señalaron que la hospitalidad de la gente, el espíritu de comunidad y hermandad y el asegurarse de que todos estaban bien fueron aspectos que ayudaron a ese cambio en su percepción. Un ejemplo de ello es el siguiente comentario: "Yesterday I took a taxi. I asked the taxi driver where was such and such and he explained to me. When I got off the taxi kept following me and then I thought, what the hell. I was a bit nervous but I stop and then the taxi driver in Spanish of course told me that I was walking the wrong way that I need to go on the opposite direction (laugh)" ("Ayer tomé un taxi. Le pregunté al taxista dónde quedaba un lugar y él me lo explicó. Cuando me bajé del taxi, el taxista me seguía y no paraba de seguirme hasta que yo me detuve un poco nervioso pensando: ' $\mathrm{Y}$ ahora qué diablos pasa', y entonces el taxista me dijo que iba en la dirección equivocada [se ríe])".

Entre las sugerencias, se señalan algunas, tales como: llegar dos o tres días antes de iniciar las clases, seguramente para tener tiempo de adaptarse; tener más horas de clase, pero también más tiempo libre; pensar en una inmersión más larga, de tres semanas, por ejemplo; hacer menos cambios en 
la agenda para organizarse mejor; realizar salidas culturales más cortas (en algunas tuvieron que viajar cuatro horas) y hacer más actividades antes de viajar a Colombia para prepararse mejor.

\section{Conclusiones}

A partir de lo señalado anteriormente con respecto al desarrollo de la experiencia y a los resultados obtenidos, podemos concluir que los cursos de inmersión en el aprendizaje de una lengua extranjera parecen ser una buena oportunidad para desarrollar la competencia comunicativa intercultural. Además de conocer sobre la "alta" y "baja" cultura colombiana, los estudiantes pudieron vivenciar la interculturalidad en la práctica de la interacción entre la cultura colombiana y la australiana y en el intercambio y comunicación con los colombianos. Sin embargo, no podemos determinar el grado de ajuste cultural logrado (superficial o profundo), tal como lo señala Fantini (2000), puesto que no es suficiente sustentación contar con las afirmaciones de los estudiantes y profesores involucrados. Si se quiere "medir" lo anterior, es necesario contar con instrumentos de recolección de datos más potentes y más confiables. Este aspecto de la medición del desarrollo de la competencia comunicativa intercultural resulta complejo y es una de las áreas en enseñanza y aprendizaje de ELE en las cuales aún hay mucha investigación empírica por realizar.

Igualmente, si tenemos en cuenta que el desarrollo de la competencia comunicativa intercultural es una decisión individual y que la experiencia cultural e intercultural de cada estudiante es única y particular, como lo es cada individuo (Fantini 200o), es necesario realizar actividades que, como se señaló en los conceptos de base, le permitan al estudiante "ejercitarse" en el "autodescubrimiento" y la percepción de hechos culturales y en su comparación no valorativa. Al parecer, las actividades realizadas antes de viajar posibilitaron que los estudiantes asumieran el reto de vivenciar la cultura colombiana y trataran de adentrarse en ella, respetando aquellas diferencias que pudieran entrar en choque con su cultura.
Sin embargo, resulta complicado pretender que las comparaciones, que naturalmente emergen, no sean valorativas.

En cuanto a la concepción de la naturaleza de "doble vía" de la competencia comunicativa intercultural (Fantini, 200o) en el caso de esta experiencia no es posible afirmar si efectivamente los estudiantes pudieron reflexionar profundamente acerca de su propia cultura, a propósito del desarrollo de la competencia en la cultura colombiana y en la lengua española. A partir de lo comentado por los estudiantes, solamente se evidenciaron algunas situaciones en las cuales establecieron comparaciones entre las dos culturas. Posiblemente, si en este momento se recolectara información con respecto al tema, se podría determinar por lo menos el grado de profundidad en las reflexiones que la experiencia suscitó.

Por otra parte, el ambiente en el cual se desarrolle la experiencia de inmersión resulta determinante para ayudar o no a desarrollar la competencia comunicativa intercultural. En los resultados obtenidos en esta inmersión, los estudiantes mencionaron haberse sentido en un ambiente cómodo y hospitalario que los había ayudado a interactuar mejor, a usar la lengua meta y a vivir una experiencia positiva, así como a cambiar puntos de vista con respecto a la lengua, el país y su gente (Fantini, 200o). Este último es, posiblemente, el aspecto en el que fue más notorio el desarrollo de la competencia comunicativa intercultural de los estudiantes. Ningún estudiante reportó haber vivido un ambiente poco hospitalario y, por lo tanto, sentirse limitado en el uso de la lengua meta o crear actitudes negativas o estereotipos.

En relación con las clases de lengua extranjera, en ellas se utiliza la lengua de manera diferente a como se usa en las situaciones cotidianas fuera del salón, en el país anfitrión, a pesar del uso de metodologías comunicativas. En este sentido, la experiencia vivida por los estudiantes australianos en los hogares de familias colombianas y con estudiantes nativos de la lengua española, les permitió adquirir conocimientos lingüísticos y culturales 
adicionales a los adquiridos en clase, desarrollar más sus habilidades de comprensión y expresión, especialmente oral, y enriquecer el léxico que se usa en situaciones comunicativas cotidianas entre jóvenes. Estos resultados corroboran lo hallado en los estudios de Levin (200I) y Berch et al. (1993), quienes demostraron que los estudiantes mejoran lingüísticamente dependiendo de la capacidad y el interés individual y del deseo de interactuar con otros y acoger la nueva cultura.

En cuanto a la efectividad de los cursos cortos frente a los intercambios en los cuales los estudiantes permanecen en el país de la lengua y cultura meta por largos periodos, según lo señalan Collentine y Freed (2004), no es posible sacar conclusiones, puesto que no se realizó ninguna comparación entre el nivel de desarrollo de la lengua y la cultura de los estudiantes australianos y el de los estudiantes en cursos más largos. Lo que sí se pudo establecer fue la efectividad del curso de inmersión, ya que, en dos semanas, los estudiantes cursaron todo un nivel y lograron adquirir las competencias establecidas por la PUJ para dicho nivel.

La sensibilización previa y el conocimiento hacia los códigos culturales de una sociedad, en este caso la colombiana, tal como se hizo antes del viaje de los estudiantes australianos al país, parece ser fundamental en experiencias de esta naturaleza, para que los estudiantes puedan interactuar adecuadamente, mejorar las habilidades comuni-

\section{Referencias}

Agray, N. (2010). Integración entre lengua y cultura. Un dilema central en la enseñanza de lenguas. Revista Innovación y Ciencia, $V$ (XVII), 29-38.

Bakalis, S., \& Joiner, T. A. (2004). Participation in tertiary study abroad programs: The role of personality. The International Journal of Educational Management, 18(5), 286-29I. cativas e interculturales y sentirse más seguros y cómodos en el desarrollo de la experiencia, tal como se señala en las corrientes metodológicas comunicativas (Nebrija, I998).

Lo anterior permite establecer que, efectivamente, el trabajo conjunto entre las instituciones y el acompañamiento a los estudiantes antes, durante y después de una experiencia de esta naturaleza es el que garantiza el éxito de esta. Solamente si se prepara y desarrolla conjuntamente la inmersión, los estudiantes pueden conocer y vivir una nueva ciudad, su cultura, su gente y su lengua de una forma positiva que les permita ir creciendo y preparándose para ser los ciudadanos del mundo que las instituciones desean ayudar a formar y que la sociedad necesita (Gardner, 20Io).

Asimismo, experiencias como la de este curso de inmersión se perciben como una buena oportunidad para estrechar los lazos de cooperación entre las instituciones, en este caso en el campo del español como lengua extranjera, y concretar las estrategias de internacionalización propuestas por las universidades (Plan Estratégico de RMIT, 20I0; y Política de Internacionalización de la PUJ, 2009).

Por último, es importante señalar que, a pesar de la imagen negativa de Colombia difundida por los medios de comunicación, este grupo de estudiantes australianos al terminar su estadía en el país se llevó una imagen completamente diferente $y$, por consiguiente, recomiendan a otros estudiantes hacer un curso de inmersión en este país.
Brecht, R. D., Davidson, D. E., \& Ginsberg, R. B. (I993). Predictors of foreign language gain during study abroad. NFLC occasional papers. Washington: National Foreign Language Center.

Collentine, J., \& Freed, B. F. (2004). Learning context and its effects on second language acquisition. Introduction. Studies in Second Language Acquisition, 26(2), I53-I7I. 
DeLoach, S., Saliba, L., Smith, V., \& Tiemann, T. (2003). Developing a global mindset through short-term study abroad: A group discussion approach. Journal of Teaching in International Business, 15(I), 37-59.

Douglas, C., \& Jones-Rikkers, C. G. (200I). Study abroad programs and American student worldmindedness: An empirical analysis. Journal of Teaching in International Business, 13(I), 55-66.

Fantini, A. (2000). A Central Concern: Developing Intercultural Competence. Recuperado de http://www.sit.edu/publications/docs/ competence.pdf.

García, J., \& Bejarano, L. G. (2008). Adquisición de una segunda lengua en estancias cortas en el extranjero: análisis actitudinal. Didáctica. Lengua y Literatura, 20, II7-I34.

Gardner, M. (2010). Plan estratégico de RMIT 2011-2015: Where Bold Hopes Can Be Realised. Recuperado de http://www.rmit.edu.au/ about/strategy.

Gudykunst, W. B. (2004). Bridging differences: effective intergroup communication (4th ed.). Thousand Oaks, CA: Sage Publications.

Hall, S. (1997). Representation. Cultural representations as signifying practice. Thousand Oaks, CA: Sage Publications.

\section{Notas}

1. Traducción hecha por las autoras de este artículo. Plan estratégico del RMIT 2011-2015: Where Bold Hopes Can Be Realised (2010). "As a university of technology and design, RMIT is not only focused in its education and research, but also committed to a philosophy of education and research founded in action and experience. This requires education and research that draws on partners and works with others to achieve our goals. [...] We must begin with our students and a commitment to transform their futures, no matter where
Jackson, J. (2010). Intercultural journeys. From study to residence abroad. New York: Palgrave Macmillan.

Kitsantas, A. (2004). Studying abroad: The role of college students' goals on the development of cross-cultural skills and global understanding. College Student Journal, 38(3), 44I-453.

Kitsantas, A., \& Meyers, J. (200I). Studying abroad: does it enhance college student cross-cultural awareness? (Educational Resources Information Center No. ED 456648$)$.

Levin, D. M. (200I). Language learners's sociocultural interaction in a study abroad context. $\mathrm{PhD}$ thesis, Indiana University. Dissertation Abstract International. 62 (2).

Lustig, M. W., \& Koester, J. (2006). Intercultural competence: interpersonal communication across cultures (5th ed.). New York: Longman.

Pontificia Universidad Javeriana. (2009). Acuerdo $N^{o}$ 448. Políticas para la Internacionalización de la Universidad. Recuperado de http://pujportal.javeriana.edu.co/portal/page/portal/ portal_version_20022010/internacionales/ resources_vi/acuerdo\%20_44 $\underline{8}$ de_ 2007.pdf

Universidad de Nebrija. (1998). Curso de metodología en la enseñanza de español como lengua extranjera. El componente cultural en los diferentes enfoques. Madrid: mimeo.

and how they begin". En http://www.rmit.edu.au/ about/strategy, recuperado en julio de 2013.

2. Acuerdo No. 448 (mayo 2007) Políticas para la Internacionalización de la Universidad. Pontificia Universidad Javeriana. En http://puj-portal.javeriana.edu.co/portal/ page/portal/PORTAL_VERSION_2009_2010/internacionales/resources_v1/ACUERDO\%20_448_de_2007. pdf, recuperado en noviembre de 2013.

3. Traducción libre de Agray, N. (2010, p. 30).

4. Traducción libre de Agray, N. (2010, p. 30). 